\title{
Grade 9 Mathematics Learners' Strategies in Solving Number-Pattern Problems
}

\author{
Erica Dorethea Spangenberg ${ }^{1 *}$, Aphane Koko Pithmajor ${ }^{1}$ \\ ${ }^{1}$ University of Johannesburg, SOUTH AFRICA
}

Received 28 January 2020 - Accepted 1 April 2020

\begin{abstract}
Many Grade 9 mathematics learners face difficulties in solving number-pattern problems due to insufficient exposure to strategies in solving such problems. The aim of this exploratory qualitative study was to investigate Grade 9 mathematics learners' strategies in solving number-pattern problems. The problem-solving conceptual framework of Singer and Voica (2013) highlighting four indicators, namely decoding, representing, processing and implementing, underpinned the study. Ninety Grade 9 learners were purposively selected from three rural schools (30 from each school) to participate. Qualitative data were collected through a written activity and semistructured one-on-one interviews. The study found that learners utilise four main strategies to solve number-pattern problems, namely (1) direct counting; (2) direct proportion; (3) recursive strategy; and (4) mental image representation. Knowledge about learners' strategies can assist teachers in their pedagogies to teach number-pattern problems. The study adds to the research on problem solving, specifically pertaining to number patterns.
\end{abstract}

Keywords: learners, mathematics, number-pattern, problem solving, strategies

\section{INTRODUCTION}

Worldwide, problem solving is infused in mathematics curricula (Liljedahl, Santos-Trigo, Malaspina, \& Bruder, 2016). In Malaysia, learners are required to develop decision-making competencies (Tambychik \& Meerah, 2010). In Singapore, problem solving is presented as a function of five interrelated components: skills, concepts, processes, attitudes, and metacognition (Anderson, 2009). In South Africa, the context of this study, the mathematics Curriculum and Assessment Policy Statements (CAPS) document for Senior Phase (SP) advocates problem solving to appreciate the physical, social and economic environment, and to develop critical thinking skills (Department of Basic Education (DBE), 2011).

However, according to Van Staden and Motsamai (2017), South African learners continue to perform poorly in mathematics throughout all grades compared to their counterparts internationally, locally and regionally. Specifically, the majority of learners in South Africa face difficulties in solving number pattern problems (Govender, 2012) According to Maluleka (2013), the greatest difficulty in working with mathematical problems is to translate real-life issues into mathematical problems. A person first need to have knowledge of the issue before trying to attempt it.

This study is situated within three Quintile 1 schools in a village in the Limpopo Province in South Africa, where most learners underperform in mathematics. Quintile 1 schools are in deep rural areas and mostly inadequately resourced in terms of teaching and learning materials, and poor infrastructure. Schools A and B are classified as underperforming schools with Grade 12 results below the benchmark of $65 \%$ for three consecutive years, while School C is a performing school with a Grade 12 pass rate of above $80 \%$ for the past number of years. Moreover, the selected schools share common features, such as poverty, absence of parental and community engagement, and a number of curriculum challenges. Mathematics teachers from these schools receive less professional training than those from suburban schools. Learners from the selected schools also share textbooks, mathematical instruments and other resources. The selected learners were 15-17 years old and had exposure to number-pattern problems as prescribed in the CAPS document for the specific grade.

According to Barbosa, Vale and Palhares (2012), an in-depth investigation of patterns can add value to the relevance of problem solving. Number-pattern problems 


\section{Contribution to the literature}

- The study adds to the research on problem solving, specifically pertaining to number patterns.

- The study informs teachers about learners' interpretations of the strategies they utilise during the different phases of problem solving, which can assist them in their pedagogies to teach number-pattern problems.

- This study is original by using the problem-solving conceptual framework of Singer and Voica (2013) as a tool to analyse the strategies learners employ in solving number-pattern problems.

involve a process of looking for numbers in a given sequence to form a pattern that allows for generalising a solution applicable to any given situation (DBE, 2011). Number-pattern activities lead to the development of problem-solving capabilities by highlighting the evaluation of specific instances, by systematically organising information, and by inferring and generalising information (Barbosa et al., 2012). Numberpattern problems also allow for the construction of mental images, which could represent the relationship between terms and their position in a sequence (Mahlobo \& Nthombela, 2014).

However, according to Boonen, Van der Schoot, Van Wesel, De Vries and Jolles (2013), solving numberpattern problems presents difficulties if learners cannot identify the relationship between the known and the unknown variable. Jupri and Drijvers (2016) confirmed that the major difficulties encountered by learners when dealing with number-pattern problems are to comprehend the mathematical vocabulary, to translate the problems into mathematical formula to present models, to answer problems presented in the model, and to deduce explanations in terms of the initial problem.

Working with number patterns is particularly difficult for learners as the patterns are often embedded in problem solving. Problem solving becomes difficult for learners when the problem is presented linguistically, since learners are required to peruse and decode the problem, symbolise the semantic formulation of the problem, and choose a solving method (Schumacher \& Fucks, 2012). Learners cannot easily identify number patterns embedded in problems. Barbosa et al. (2012) stated that students tend to use numeric rather than visual methods and experience several difficulties when solving pattern exploration issues, particularly when generalising remote values. Usually, learners have trouble in problem solving when trying to translate algebraic problems into mathematical equations (Ahmad, Tarmizi, \& Mawawi, 2010).Various researchers recommended further studies in problem solving. Boonen et al. (2013) suggested studies focusing on teaching learners to construct mental models analytically during problem solving. Duru, Peker, Bozkurt, Akgün, and Bayrakdar (2011) advocated the need for teachers to be informed about various strategies to solve problems in mathematics, and to understand learner difficulties in order to implement these strategies. Jan and Rodrigues (2012) proposed studies to determine the variables affecting the capacity of learners to understand mathematical problems in order to establish suitable teaching approaches to solve problems meaningfully.

The significance of this study in terms of academic value is that it expands on the existing problem solving (PS) conceptual framework of Singer and Voica (2013), which may be used for further research pertaining to the development and maintenance of effective strategies to solve problems involving number patterns. The study also contributes to practice by providing information about learners' strategies to solve problems, which could inform teachers about learners' interpretations of the strategies they utilise during the different phases of problem solving to assist them in their pedagogies to teach number-pattern problems.

\section{PURPOSE AND RESEARCH QUESTION}

The purpose of this study is to investigate Grade 9 mathematics learners' strategies in solving numberpattern problems. The following research question is interrogated: What strategies do Grade 9 mathematics learners use in solving number-pattern problems?

\section{LITERATURE OVERVIEW}

The section situates number patterns in problem solving and focuses on strategies to solve problems and the PS conceptual framework of Singer and Voica (2013).

\section{Number Patterns in Problem Solving}

Peters (2011) defined mathematics as the science of finding patterns. Patterns are powerful tools in mathematics and can suggest several approaches, namely knowledge of counting numbers, recognition of numbers, and relating numbers to the identification of pattern. Number-pattern tasks are problems that involve a number sequence. Recognising number patterns, as related to problem solving in this study, involves a process of looking out for numbers in a given sequence and forming a pattern, which allows the problem solver to generalise a solution that can be applied in every given situation.

The learning of patterns and sequences starts in South Africa in the foundation phase where learners engage with both number patterns (e.g. skip counting) and 
geometric patterns (e.g. pictures). In the intermediate and SP phases number patterns in mathematics are about recognising, describing, and working with numerical and non-numerical patterns and where learners are expected to make predictions and justify their reasoning in their engagement with number pattern problems (DBE, 2011).

According to the SP CAPS, there are two kinds of patterns in mathematics, namely number patterns and geometric patterns (DBE, 2011). With number patterns, learners are given a sequence of numbers and they have to identify a pattern or relationship between consecutive terms in order to extend the pattern. Examples of number patterns are $-2,-5,-8,-11 \ldots$ or $3,7,11,15,19 \ldots$ where the second term depends on what happens to the first term, and the third term depends on the second term. Liljedahl (2004) argued that number patterns contain numbers, which cannot be transferred to

non-numeric patterns without loss of some crucial property of the pattern ... For example, the pattern $1,2,3,4,3,2,1$ is transferable to a b c d c b a; and $1,2,1,1,2,1,1,1,2 \ldots$ can be transferred to $\mathrm{a} b \mathrm{a} a \mathrm{~b}$ a a $\mathrm{a} b .$. without loss of the nature of the pattern. Thus, these two patterns cannot be defined as number patterns (p. 4).

Conversely, geometric patterns are number patterns represented diagrammatically. The diagrammatic representation reveals the structure of the number patterns (DBE, 2011), for example, flower patterns or matchstick patterns. Such patterns usually require some form of generalisation of patterns, usually in terms of algebraic symbols. Moreover, there is a variety of different number patterns in mathematics, including linear or arithmetic sequences and quadratic sequences (DBE, 2011).

A number pattern or geometric pattern is a linear sequence if each number is obtained by adding a constant increment to the previous number; while a quadratic sequence is a sequence of numbers in which the second difference between each consecutive term differs by the same amount, e.g., $-3 ; 8 ; 23 ; 42 ; 65$. To confirm that the sequence is quadratic, the second difference must be found. According to the CAPS, patterns in mathematics are about describing and working with numerical and non-numerical patterns (DBE, 2011). Patterns may be represented in concrete, visual words or symbolic forms using shapes, pictures and colours.

\section{Strategies to Solve Problems}

Problem solving is a skill contributing to a process, which involves thinking, reasoning, interpreting, analysing, predicting, evaluating and finding strategies to solve a problem (Anderson, 2009). Learners are required to understand a problem in a situation, to identify keywords to help to solve the problem, to create strategies to solve the problem, and to apply strategies to arrive at the solution. Schumacher and Fuchs (2012) pointed out that mathematical problem-solving tasks are presented linguistically and do not require learners to do straightforward calculations. Learners have to read with understanding and be able to interpret the problem, represent the model or equation from the written statement, and choose a solution strategy.

Strategies are groupings of mental actions designed to solve a problem (Biddlecomb \& Carr, 2011) and offer learners with situations to explore certain elements, principles, and ideas embedded in mathematics topics (Killen, 2007). Specifically, Garcia-Cruz and Martinon (1997) identified three primary strategies: counting strategy, direct proportional strategy, and recursive approach. Ibrahim and Rebello (2013) added mental representation and indicated that learners operate at three levels of mental representation, namely propositional, mental images and mental model representation.

A counting strategy comprises the counting of the elements of a particular number or figural term in a pattern (Barbosa et al., 2012). For example, to add $4+3$, learners start with the 4 , and then count up: $5,6,7$. When adding negative numbers to negative number (counting backwards), for example, $-4+(-2)$, learners have to disregard the plus sign and recognise that they are subtracting the first negative number by the second negative number, thus negative four minus two. They have to start at -4 , then count 2 more backwards, thus subtracting. Their response will then be -6 .

A direct proportion strategy employs a term as a row to form a larger row by applying multiples of the original term, thus using a portion as a unit to construct a larger unit using multiples of the units (Lannin, Barker, \& Townsend, 2006). For example, if learners need six white beads and one black bead to make one flower, by applying the direct proportion strategy, they will say for three flowers, there will be $6 \times 3=18$ white beads and 1 x $3=3$ black beads. The learners count how many white beads are needed for the second flower, and look for a rule that would work, namely multiplying 6 , which is the number of white beads for one flower, multiplying by the number of flowers.

A recursive technique is the connection between successive independent variable values in the scenario (Lannin et al., 2006). Learners select a recursive strategy if they want to determine a general rule based on an understanding of a relationship that occurs in the situation. For example: To find a general rule for the following pattern $5 ; 13 ; 21 ; 29 ; 37 \ldots .$. , learners will first find the common difference, which is 8 , and multiply with the $n^{\text {th }}$ term, $n \times(8)=8 n$ where $n$ represents the number of term in the sequence and 8 indicates the common difference. For the first term $\left(\mathrm{T}_{1}\right)$, learners will 


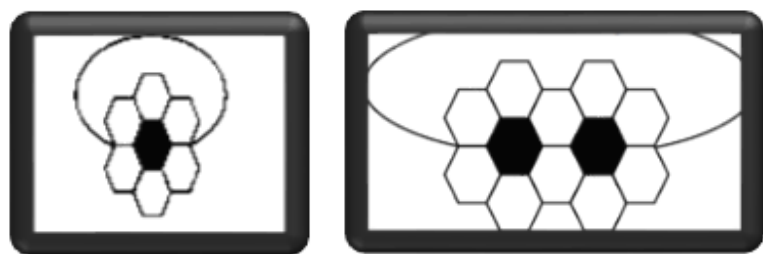

Figure 1. Necklaces made with beads

check whether $T_{1}=1 \times 8=8$ gives them the first term of the sequence, which is 5 , which is not true in this case. Thus, they need to subtract 3 to give 5 . For the second term $\left(T_{2}\right), T_{2}=2 \times 8=16$, which does not give 13. Thus, they should also subtract 3 to give 13 . Therefore, the recursive rule will be $\mathrm{T}_{n}=8 n-3$.

Ibrahim and Rebello (2013) defined a mental image representation as a diagrammatic representation or model. Mental models assist in connecting the mathematical and visual aspects of a task to ensure better understanding of the problem. Although learners may be aware of the relationship among the white and the black beads to make a flower as illustrated in Figure 1 and may recognise the applicability of the qualitative method (taking the number of whites beads and the number of flower) to solve the problem, they may prefer to manipulate equations. The learners may see these flowers as a disjoint unit that does not make a linear pattern, thus handling visual representations in a mental image in isolation. When learners have to interpret the visual pattern, they need to formulate a description referring to each flower as a joint unit to form a linear pattern. For example, each flower shares two white beads to form a complete flower. Four white beads have to be added to the end of each prior flower to create a new flower and another two beads to form a complete flower. Thus, the general rule will be $\mathrm{T}_{n}=4 n+2$, where the variable $n$ represents the number of flowers.

\section{Problem-Solving (PS) Conceptual Framework of Singer and Voica (2013)}

The PS conceptual framework of Singer and Voica (2013) underpins the study, which is designed to assist in reading a problem with understanding by identifying keywords that could help in solving the problem. The solution process is effective if learners can interpret the solution in relation to the wording of the given problem. Learners should understand the solution from the original statement within which the problem is given by connecting the solution of the problem with the initial problem statement. The PS conceptual framework describes four linear phases to move from the initial information on the given text to the correct solution, namely decoding, representing, processing, and implementing.

During the decoding phase (DP), learners should interpret the given statement and identify the keywords in solving the problem (Clement, 2008). For this study, participants reached the DP if they could use correct mathematical operators in order to extend the pattern to the next number, thus, identifying the mathematical operators that would assist them in ascertaining the type of number pattern involved in a given problem.

During the representing phase (RP), learners must represent the problem in the text by using a suitable mathematical image (Singer \& Voica, 2013), such as drawing, words, symbols, graphs or table values. Participants in this study reached the RP if they could explore possible situations through drawings, and find the connection between the data and the unknown in the given problem statement to produce a mental model (with or without visual support) suggested by the problem.

During the processing phase (PP), learners should use their mathematical understanding to identify the correct mathematical model that is relevant to the problem, such as an equation, a system, the steps of a graphical representation or various computing algorithms (Singer \& Voica, 2013). For this study, participants reached the PP if they could find the general formulae of the sequence, find the relevant equation of the $n^{\text {th }}$ term of the sequence by using the correct mathematical operation sign, or transpose the text into an equation.

Lastly, the implementing phase (IP) refers to the application of methods specific to a mathematical model representing the problem, with the purpose to solve the problem (Singer \& Voica, 2013). For this study, participants reached the IP if they could implement the mathematical model suggested by the problem to find any $n^{\text {th }}$ term of the sequence.

\section{METHODOLOGY}

\section{Research Design, Sampling and Research Instruments}

This study adopted an exploratory qualitative approach and was conducted in three rural schools from a district in Limpopo, South Africa. Thirty Grade 9 mathematics learners from each of these schools, in total 90 , were randomly selected to complete a written activity on number-pattern problems on a voluntarily basis. Thereafter, individual one-on-one semi-structured interviews were conducted with three of them, one from each school, based on the solutions in the written activity, namely: No answer (Participant A15); irrelevant strategy (Participant B2); and appropriate specific strategy (Participant C5).

\section{Design of the Written Activity}

The written activity consisted of two questions. For Question 1, participants had to describe a decreasing pattern by indicating that the pattern became small, or shrank, by a constant amount each time. For Question 2, participants were expected to describe an increasing 
Table 1. Number sequence

\begin{tabular}{llllllllll}
\hline$x$ & 1 & 2 & 3 & 4 & 5 & 6 & 7 & 8 & $\mathrm{~N}$ \\
\hline$f(x)$ & -2 & -5 & -8 & -11 & -14 & & & \\
\hline
\end{tabular}

geometric pattern, and to relate the concepts of a linear function to the geometric diagram. The two questions are presented next.

\section{Question 1}

Complete Table 1 by indicating the next terms $(f(x))$ of the sequence. If the sequence is continued to $n^{\text {th }}$ term, please write the general formula for the $n^{\text {th }}$ term. Explain your answer.

\section{Question 2}

Joana likes to make necklaces using flowers. She uses white beads for the petals and black beads for the centre of each flower. Figure 1 shows a necklace with one flower and a necklace with two flowers, both made by her.

- How many white and black beads will Joana need to make a necklace with three flowers? Explain your answer. Make a neat diagram with three flowers.

- How many flowers will Joana be able to make if she uses 102 white beads? Explain your answer.

The written responses of the participants were marked and the data were analysed according to levels of engagement for the four phases of the PS conceptual framework using predetermined indicators as shown in Table 2.

\section{Design of Interviews}

In Stage 2, one-on-one semi-structured interviews were conducted with the three selected participants (Participants A15, B2 and C5) to verify the strategies used in the written activity and to ensure that the strategies were correctly interpreted. The questions asked were:

1. Let us look at Question 1. What type of pattern is it and why?

2. How did you complete the sequence in Question 1 ? Explain your strategy.

3. How did you get your general formula?

4. When working with Question 2; how did you identify the keywords to solve the problem?

5. How did you make a necklace with three flowers?

6. How many flowers will Joana be able to make if she uses 102 white beads? Explain your answer.

7. Can you come up with a general formula for this problem?

8. How do you determine if the formula used is correct? Is there anything else you want to tell me with regard to better understanding of numberpattern problems?

The interview recordings were transcribed and were coded according to the pre-determined indicators in Table 2.

\section{Trustworthiness}

Triangulation by analysing and comparing results from two data sources, namely documents and interviews, ensured trustworthiness. Specifically, multiple qualitative data collection instruments were used. To ensure credibility, audiotaped interviews were transcribed and the inconsistent data were carefully checked and examined. Member checking ensured the rectification of inconsistent data and validation of emerging findings. However, it was difficult to validate the emerging findings, as participants could have reached a particular phase of the PS conceptual framework in their minds, but chose not to write down the solution they arrived at for not trusting that the solution is accurate. To ensure transferability, a thorough literature review and a dense description of the methodology were provided for replication of the study. A literature control and verbatim quotes were also provided. To ascertain dependability, the researcher continued interviewing participants until datasaturation was reached. Confirmability was established by external audits. Experienced researchers advised regarding the setting and assessment of the written activity and interview questions. To analyse the data, we coded each solution to the written activities and each comment from the transcripts. These codes were demarcated as being a phrase, sentence or group of successive sentences that expressed a particular concept. On first reading, the written activities and the transcripts were broken down into codes, and thereafter a further reading determined which pre-determined indicators, based on the PS framework of Singer and Voica (2013), each code was classified as. This coding was done three times by the first author, and twice by the second author, followed by an agreement upon these codes, to establish inter-rater reliability and to reach data saturation in the coding. Ethical clearance was obtained from the university overseeing the study (Ethical clearance number 2017-029) and the Department of Education in Limpopo and all ethical measures were adhered to.

\section{DATA ANALYSIS}

Participants' levels of engagement were established by coding the participants' actions they displayed in their responses to the written activity according to the pre-determined indicators. Figure 2 shows solutions to 
Table 2. Indicators for analysing the levels of engagement for the four phases of the PS conceptual framework

Decoding phase (DP)

Indicator Levels of engagement

DP3 Show an understanding of the level of engagement in the DP accurately, appropriately and flexibly by interpreting, recognising and expanding a number pattern, and related numbers and operations with the problem statement.

DP2 Show an acceptable level of engagement in the DP with minor errors, but can not show knowledge of how to use the mathematical operation of subtraction.

DP1 Display some insight into decoding, but with major mathematical errors by making major mistakes when interpreting the mathematical structure and a numerical pattern. Concentrate only on the relationship between a single pair of beads (white beads) and use it as a general rule.

DP0 Leave blank spaces, do not respond to the question, or show no level of engagement in the DP.

Representing phase (RP)

RP3 Focus on the comprehension of the situation, as well as the mathematics concepts in the activity. Having a mental model prioritising a qualitative approach, such as an explanation of how to get the next term of the sequence to complete the table value and to draw the correct diagram.

RP2 Show an awareness of the relationship among the flowers and the beads, and can mention the previous term and the next term. Recognise the applicability of the qualitative method to solve the problem. Provide reasons for the problem even if failing to handle the mathematical part of the problem. For example, recognising the common difference between consecutive numbers, but incorrectly using it to determine the next term. Although reasoning about the number of beads and the flower, an incorrect diagram is used.

RP1 Tend to use definitions and handle mathematical formulations in a rote and mechanical manner. Show no evidence of understanding of the underlying concepts of a number pattern. Manipulate equations by directly applying multiplication of the common difference when the pattern increases.

RP0 Leave blank spaces or do not respond to the question.

\section{Processing phase (PP)}

PP3 Construct a mathematical model and use it to show how the number of a previous term depends on the number of the next term.

PP2 Create a mathematical model, with minor errors in finding the term of the sequence. Make application errors by thinking that $n$ is the term that follows the previous term and apply numerical expressions instead of using an algebraic expression.

PP1 Create an incorrect formula to generalise the sequence. Aply the formula of the common difference that is $T_{2}-T_{1} ; T_{3}-$ $\mathrm{T}_{2} \ldots$ as a general formula.

PP0 Leave blank spaces or do not respond to the question.

Implementation phase (IP)

IP3 Apply techniques that are specific to the found mathematical model. Implement the correct general rule to obtain the correct solution.

IP2 Implement the solution in a manner that addresses the problem statement, but ignore relevant contextual factors. Indicate that the rule to find the number of white beads is $\mathrm{T}_{n}=6 n$.

IP1 Implement the solution in a manner that does not directly address the problem statement. Are aware of the common difference between consecutive numbers, but conduct the wrong mathematical model and use a direct proportional model to implement the solution, e.g. $\mathrm{T}_{n}=-3 n$.

IP0 Leave blank spaces or do not respond to the question.

Table 3. Participants' levels of engagement according to the phases of the PS conceptual framework in the written activity

\begin{tabular}{lcccc}
\hline Level & \multicolumn{4}{c}{ Phases } \\
\cline { 2 - 5 } & Decoding (DP) & Representing (RP) & Processing (PP) & Implementing (IP) \\
\hline $\mathbf{0}$ (No response) & 20 & 20 & 20 & 20 \\
$\mathbf{1}$ & 30 & 28 & 25 & 22 \\
$\mathbf{2}$ & 19 & 16 & 13 & 11 \\
$\mathbf{3}$ & 21 & 15 & 11 & 9 \\
\hline
\end{tabular}

the questions in the written activity of Participant A4. The solutions were grouped within the codes assigned to them according to the four phases of the PS conceptual framework.

Table 3 illustrates the number of participants showing levels of engagement according to the four phases of the PS conceptual framework.
The participants' levels of engagement according to the four phases of the PS conceptual framework deduced from the interviews were also coded using the predetermined indicators presented in Table 4. 


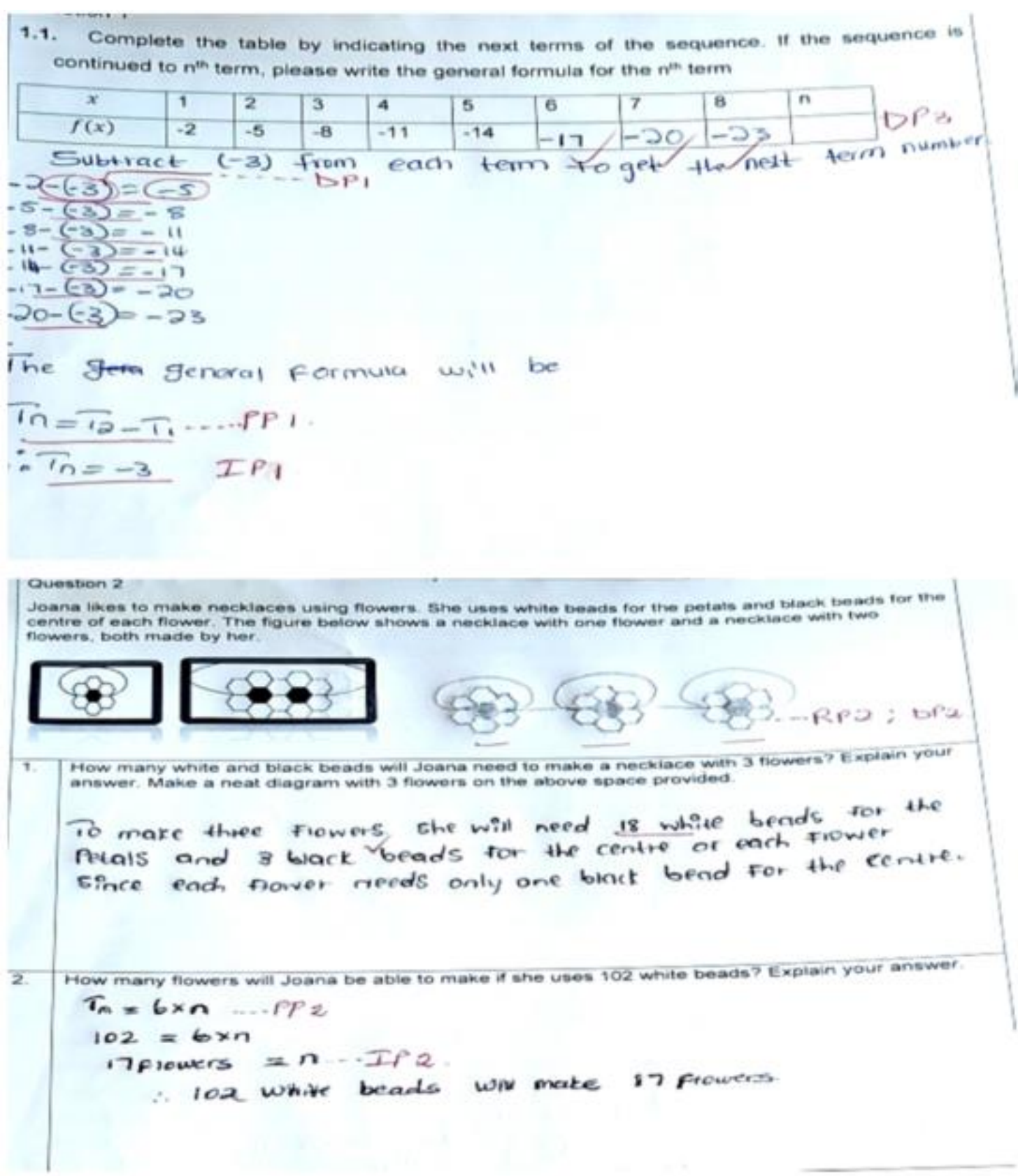

Figure 2. Participant A4's solutions to the written activity according to the four phases of the PS conceptual framework

Table 4. Participants' levels of engagement according to the phases of the PS conceptual framework in the interviews

\begin{tabular}{lccccc}
\hline Phases & Indicators & \multicolumn{3}{c}{ Number of responses } & Total number \\
of responses
\end{tabular}


Table 5. Participant C5's comments from the transcript according to the four phases of the PS conceptual framework

\begin{tabular}{|c|c|c|c|}
\hline Decoding & Representing & Processing & Implementing \\
\hline $\begin{array}{l}\text { The pattern has a } \\
\text { common difference of }-3 \text {; } \\
\text { therefore, I used the } \\
\text { common difference to get } \\
\text { the next term, which will } \\
\text { be } \\
-2-3=-5 \text {, then continue } \\
\text { like that until I arrive to } \\
\text { the nth term. For the } n^{\text {th }} \\
\text { term I used the general } \\
\text { formula (DP3) }\end{array}$ & $\begin{array}{l}\text { Each flower must be a joint unit to } \\
\text { form a linear pattern. Each flower } \\
\text { shares two white beads to have a } \\
\text { complete flower, four white beads } \\
\text { were added to the end of each prior } \\
\text { flower to create a new flower, and } \\
\text { then added two beads to give me a } \\
\text { complete flower (RP3) } \\
n \times(-3)=-3 n \text {; } n \text { represents the } \\
\text { number of term in the sequence and - } \\
3 \text { is the common difference. } \\
\text { Term } 1=1 \times(-3)=-3 \\
\text { This does not give }-2 \text {, which mean I } \\
\text { must add } 1 \\
\text { Term } 2=2 \times(-3)=-6 \\
\text { This does not give me }-5 \text { as the } \\
\text { second term I must add } 1 \text {; therefore I } \\
\left.\text { will have } T_{n}=-3 n+1 \text { (RP3 }\right)\end{array}$ & $\begin{array}{l}\text { My general rule will be } \mathrm{T}_{n}= \\
4 n+2 \text { the variable } \mathrm{n} \\
\text { represents the number of } \\
\text { flowers (PP3) } \\
n \times(-3)=-3 n ; n \text { represents the } \\
\text { number of term in the } \\
\text { sequence and }-3 \text { is the } \\
\text { common difference. } \\
\text { Term } 1=1 \times(-3) \\
=-3 \\
\text { This does not give }-2, \text { which } \\
\text { mean I must add } 1 \\
\text { Term } 2=2 \times(-3) \\
=-6 \\
\text { This does not give me }-5 \text { as } \\
\text { the second term } \mathrm{I} \text { must add } 1 \text {; } \\
\text { therefore I will have } \mathrm{T}_{n}=-3 n \\
+1 \text { (PP3) }\end{array}$ & $\begin{array}{l}\text { I will substitute } 102 \text { white } \\
\text { beads into my formula (IP3) } \\
\mathrm{n} \times(-3)=-3 \mathrm{n} \text {; } \mathrm{n} \text { represents the } \\
\text { number of term in the } \\
\text { sequence and }-3 \text { is the common } \\
\text { difference. } \\
\text { Term } 1=1 \times(-3) \\
=-3 \\
\text { This does not give }-2 \text {, which } \\
\text { mean I must add } 1 \\
\text { Term } 2=2 \times(-3) \\
=-6 \\
\text { This does not give me }-5 \text { as the } \\
\text { second term I must add } 1 ; \\
\text { therefore I will have Tn }=-3 n+ \\
1 \text { (IP3*) }\end{array}$ \\
\hline
\end{tabular}

[*] denotes a phrase extracted to be coded across the four phases of the PS conceptual framework.

Table 5 shows comments from the transcript of Participant C5, grouped within the codes assigned to them according to the four phases of the PS conceptual framework.

As can be noticed in Table 5, there were comments extracted, from within a coded comment, to be coded in another pre-determined indicator. Next, we discuss what the data analysis illuminates.

\section{RESULTS AND DISCUSSION}

\section{Written Activity}

According to Singer and Voica (2013), the problemsolving process is completed if the problem solver can progress through all four linear phases from the initial information on the given text to the correct solution, namely decoding, representing, processing, and implementing. However, from participants' written activities, it was found that many participants were not able to complete the cycle by moving to DP3, RP3, PP3 and IP3. While nine participants were able to engage in all phases, only four of them focused on comprehension of the situation, as well as the mathematics concepts. The other five participants could provide reasons for the problem, but they failed to handle the mathematical part of the problem.

Twenty of the 90 participants (22.2\%) did not respond to any question in the written activity. A further $12.2 \%$ (11 out of 90 ), 23.3\% (21 out of 90 ) and 31.1\% (28 out of 90) participants left blank spaces in the RP, PP and IP respectively. Thus, almost a third of the participants did not progress through all the phases of the PS conceptual framework by moving from the wording in the problem to the interpretation of its solution and connecting the solution with its problem statement. This finding aligns with Jupri and Drijvers (2016) noting that the major difficulties encountered by learners are to comprehend the mathematical vocabulary, to translate the problems into mathematical formula to present models, to answer problems presented in the model, and to deduce explanations in terms of the initial problem.

A third of the participants ( 30 out of $90 ; 33.3 \%$ ) could only engage on the DP1, followed by 28 of the 90 participants $(31.1 \%)$ on the RP1, 25 of the 90 participants (27.8\%) on the PP1, and 22 of the 90 participants $(24.4 \%)$ on the IP1. Thus, less than a quarter of the participants could move from the DP to the IP at a Level 1, which imply that they implemented the solution in a manner that did not directly address the problem statement. This finding aligns with Jurdak and El Mouhayar (2014) revealing that learners' level of reasoning for near generalisation is higher than that for the far generalisation when solving number patterns.

Less than a quarter of the participants (19 out of 90; $21.1 \%$ ), could engage on the DP2. Thus, most participants could not fully interpret the given statement or identify the keywords in solving the problem, as expected at the DP according to Clement (2008). Only 16 of the 90 participants $(17.8 \%)$ were able to engage on the RP2, 13 of the 90 participants $(14.4 \%)$ reached the PP2, and 11 of the 90 participants (12.2\%) achieved the IP2. Participants who reached all the phases of the PS conceptual framework decreased with $12.2 \%$ from Level 1 to Level 2. This finding suggests that the participants implemented the solution in a manner that addressed the problem statement, but ignored relevant contextual factors. Although the participants translated information from a linguistic to a visual format, they did not necessarily use the visual representation to generate a numerical solution. This finding is similar to Ibrahim and Rebello (2013) revealing that learners, who use a 
mental image, fail to translate information from the task presented with a symbolic format into a visual format for solving a problem.

Also, slightly less than a quarter of the participants (21 out of $90 ; 23.3 \%$ ), could engage on the DP3, followed by 15 of the 90 participants $(16.7 \%)$ on the RP3, 11 of the 90 participants $(12.2 \%)$ on the PP3, and 9 of the 90 participants $(10 \%)$ on the IP3. Almost the same percentage of participants could reach all the phases of the PS conceptual framework at Level 3 as at Level 2, which could indicate the participants applied techniques that were specific to the found mathematical model during implementation. Güner, Ersoy and Temiz (2013) acknowledged that for the development of algebraic thinking in mathematics, it is important for learners to have knowledge about how to construct patterns, to use cognitive processes and to think in these constructions. Most participants whose strategies were fragmented during the RP and PP when solving the number-pattern problem, recalled prior knowledge that was within the context of the problem. However, some elements of their solution strategies were incorrectly manipulated, which were caused by incorrect representation and processing of the problem. When representing their strategies, most participants confused the roles of the independent and dependent variables. Thus, they had an incorrect understanding of positive and negative numbers when describing the rule and justifying it.

Less than a half of the participants (43 out of 90) whose strategies were inappropriate to the numberpattern problem, failed to implement the mathematical model to obtain the correct answer. Although they had a mathematical model of the described situation, it was not entirely satisfactory for the problem text. This finding differs from Ibrahim and Rebello (2013) claiming that mental models should assist in connecting the mathematical and visual aspects of a task to ensure better understanding of the problem. Only 12 out of 90 participants, whose implementation was appropriate to number-pattern problems, could recall and manipulate number-patterns strategies that were relevant to the context of the problem. Those participants were developing an own understanding through meaningful decoding, representation and processing that allowed them to deeply understand the concepts and processed what was already known about the problem. They could use the model as a building block to implement the solution and their understanding of the given problem was at an advanced level. They were able to move beyond the data, as they were able to generate and predict the number patterns. Thus, their written activities showed that they had a deeper understanding of mathematical structures and mathematical concepts related to number patterns.

\section{Semi-Structured Interviews}

Eleven of 31 responses (35.5\%) indicated that participants had reached the DP. Seven of 33 responses (21.2\%) showed evidence of moving to the RP and PP, and 6 of 33 responses (18.2\%) implied some level of engagement at the IP.

\section{Decoding}

Slightly more than a third of the responses (11 out of $31 ; 35.5 \%$ ) regarding strategies used in solving numberpattern problems was evident on the DP. According to Clement (2008), a problem solver reach the DP if he/she could interpret the given statement. All three participants used mathematical operators correctly and were able to find the relationships among the data and the operators. They also showed an understanding of position-to-term relationship. However, only Participant C5 was able to proceed to the far position using the general rule. Participant C5 showed an understating of decoding accurately, appropriately, and flexibly by remarking that each flower shares two white beads to have a complete flower; four white beads were added to the end of each prior flower to create a new flower.

Participants A15 and B2 could show an understanding of decoding by finding a pattern to discover terms in the near position using the counting strategy. As Participant A15 was completing the sequence from the table, he/she mentioned, "I am counting the numbers from the sequence by subtracting 3 up until I arrive to the $n^{\text {th }}$ term". Participant B2 stated:

The sequence goes down and we called it a decreasing pattern and we represent the common difference between consecutive numbers by the variable $\mathrm{d}$; I have calculated the common difference (d) between consecutive numbers, which is -3 . For example, $\mathrm{T}_{2}-\mathrm{T}_{1}=\mathrm{d} ;-5-(-2)=-3$; $\mathrm{T}_{3}-\mathrm{T}_{2}=\mathrm{d}$ the common difference between two consecutive terms is -3 , and then I subtracted the first term by -3 to get the next term.

Participants A15 and B2 applied direct proportion to determine the term in the far position. They could employ a term as a row to form a larger row by applying multiples of the original term, thus using a portion as a unit to construct a larger unit using multiples of the units, as indicated by Lannin, Barker, and Townsend (2006). However, their strategy was incorrect for the given problem. Both participants lacked an understanding of linear pattern for far generalisation during decoding. These participants could not identify how each figure and number in the pattern differed from the previous ones as the pattern increased. In their description of the number patterns for the far position, both participants did not recognise that each term had a numeric value. They were unable to demonstrate a way of establishing a general formula of the $n^{\text {th }}$ term of the 
sequence number in a table form. This finding correspond with Barbosa et al. (2012) who disclosed that in solving problems involving pattern exploration, learners encountered several difficulties, particularly when they had to generalise for remote values. These participants accomplished better outcomes in problems of near generalisation than in far-reaching generalisation. Participants A15 and B2 began using the counting approach, although they moved to a direct proportion model for the far-reaching generalisation, which could imply that they were focusing on calculating the number of components of a shape or configuring to calculate the expected drawing a shape.

\section{Representing}

Less than a quarter of the responses (7 out of 31 ; $22.6 \%$ ) on strategies used in solving number-pattern problems, was on the RP. This finding is similar to Singer and Voica (2013) indicating that learners must be able to represent the problem in the text by using a suitable mathematical image, such as drawing, words, symbols, graphs or table values. Participant A15 focused on given or apparent information or prioritised the manipulation of equations with rote memorisation or pattern matching of information. Participant A15 mentioned, "I am weak in mathematics and poor in visualisation to see a pattern, but now as I am speaking to you I see light". Participant A15 could provide reasons for the problem even though he/she failed to handle the mathematical part of the problem by mentioning, " 6 white beads and one black bead make one flower therefore for 3 flowers will be $(6 \mathrm{x}$ 3 = 18 beads)". The mathematical structure of the sequence was not properly represented. Participant A15 interpreted each flower as a disjoint unit and therefore used the direct proportional strategy. This finding does not correspond with Barbosa et al. (2012) claiming that visual patterns enable learners to relate number contexts with visual contexts, which enhance their understanding of the meaning of numbers and variables. Although Participant A15 had a visual pattern of the situation, it could be that he/she was unable to relate number contexts with visual contexts and, therefore, did not make a final adjustment based on the context of the problem.

Participant B2 mentioned, "The sequence increases by 6 white beads in ever flower, but I don't know the type of pattern". Participant B2 understood how the subsequent terms were obtained, but was unable to establish the rule for the general term. Thus, both Participants A15 and B2 had a mental image of the problem. Although they demonstrated a diagrammatic representation (flowers), it could be that it did not link to the mathematical formulations used. This finding corresponds with Becker and Rivera (2005) revealing that variables used simply as non-significant placeholders show a lack of representation. Although Participants A15 and B2 could count number of beads from the sequence, it may be that they made no sense of what the $n^{\text {th }}$ term in the linear pattern represented, nor what the variable in the linear pattern represented. While they might have been aware of the relationship among the three flowers and the beads and could recognise the applicability of the qualitative method to solve the problem, they could have preferred manipulating equations.

When Participant C5 was asked what type of pattern is presented in the sequence, he/she answered that: "the pattern is an increasing linear pattern they sometimes call it arithmetic sequence," and indicated:

Each flower must be a joint unit to form a linear pattern. Each flower shares two white beads to have a complete flower, four white beads were added to the end of each prior flower to create a new flower, and then added two beads to give me a complete flower, therefore my general rule will be $T_{n}=4 n+2$ the variable $n$ represent the number of flowers.

Participant C5's response was categorised as having a mental model, referring to his/her ability to conduct high-level reasoning. Thus, Participant C5 could operate at three levels of mental representation, namely propositional, mental images and mental model representation, as suggested by Ibrahim and Rebello (2013). Participant C5 focused on comprehension of the situation and highlighted the mathematics concepts in the activity. Before dealing with equations he/she was included a diagrammatic representation in the solution of the problem. This finding corresponds with Ibrahim and Rebello (2013) acknowledging that the construction of the mental model provides a means of linking the mathematical and visual aspects of the task and enables interpretation and understanding.

\section{Processing}

Less than a quarter of the responses (7 out of 31 ; $22.6 \%$ ) on strategies used in solving number-pattern problems, was on the PP. According to Singer and Voica (2013), learners should be able to use their mathematical understanding to identify the correct mathematical model that is relevant to the problem, such as an equation, a system, the steps of a graphical representation or various computing algorithms. When asked to complete number patterns on a table until the $n^{\text {th }}$ term, participant A15 responded: "I counted the numbers from the sequence by subtracting 3 up until I arrive to the $n^{\text {th }}$ term, I think $n$ will be a number that follow the previous number in the sequence". Participant A15 used direct counting to solve near generalisation for the given number patterns. According to Barbosa et al. (2012), a counting strategy comprises the counting of the elements of a particular number or figural term in a pattern. For far generalisation, 
Participant A15 claimed that "the general formula will be $\mathrm{T}_{n}=-3 \times n$ ". This finding corresponds with Barbosa et al. (2012, p. 291) arguing that learners who are "not able to find adequate explicit rules, reveal difficulties in finding a functional relationship and make many mistakes, such as the application of a direct proportion model when it is not applicable". By using the counting strategy, Participant A15 might have used direct proportion because he/she found it difficult to produce a mathematical model, specifically where far generalisation was involved.

\section{Participant B2 mentioned:}

I found that the pattern decreases by 3 less than before. I tried the different possibilities, for example, $n-3,-3 n, 3 \times n$ and trying to add other digits that can give the first -2 . I then substituted $n$ by $1 ; 2 ; 3$ so that it can give me the pattern of the sequence. Then I found the algebraic expression for this kind of pattern then I choice $-3 n$. Therefore, $I$ have $T_{n}=-3 n+\ldots$.

Participant B2 could write some general formulas, but did not focus on the relationship between the term and the value of the term, nor did he/she check the accuracy of formulas by comparing the number and value of the step. Thus, Participant B2 was unable to find the general formula and failed to elaborate clearly on how he/she arrived at the rules used in the written activity. This finding is similar to Becker and Rivera (2005) claiming that learners who are unable to provide the general formulae tend to start with numerical strategies and lack the flexibility to try other approaches and see possible connections between different forms of representation and processing strategies. It could be that Participant B2 only knew the letter $n$ represented a number, whereas Participant A15 took it to mean the previous term, which imply that he/she might have been unaware of the meaning of $n$ as used in the symbolic rule.

Participant C5 responded:

$\mathrm{n} \times(-3)=-3 \mathrm{n}$; $\mathrm{n}$ represents the number of term in the sequence and -3 is the common difference. Term $1=1 \times(-3)=$, this does not give -2 which mean I must add 1 . Term $2=2 \times(-3)=-6$, this does not give me -5 as the second term I must add 1; therefore, I will have $T_{n}=-3 n+1$.

Participant C5 showed competence on producing a mathematical model during the PP by demonstrating a way of establishing a general formula for the $n^{\text {th }}$ term of the sequence number by drawing a logical conclusion. Participant A15 preferred to use a direct proportional strategy far generalisation, while Participant B2 tried to use guess and check for far generalisation. This guessing and checking strategy took the form of scribbling down the algebraic expressions. Participant B2 tested and adjusted the number in order to fit the expression to the pattern. This finding concurs with Becker and Rivera (2005) claiming that learners who are unable to provide the general formulae tend to start with numerical strategies. They lack the flexibility to try other approaches and see possible connections between different forms of representation and processing strategies.

\section{Implementing}

The phase with the least responses (6 out of 31 ; $19.46 \%)$ on strategies used in solving number-pattern problems was the IP, which could be because most of the participants had not reached this phase. This finding contradicts Singer and Voica (2013) arguing that a problem solver should be able to apply methods specific to a mathematical model representing the problem, with the purpose to solve the problem. Participant A15 implemented the solution in a manner that addressed the problem statement, but ignored relevant contextual factors. When asked to use the mathematical model to calculate the number of flowers Joana will need if she uses 102 white beads, Participant A15 mentioned, "6 white beads and one black bead make one flower. Therefore, for 3 flowers, it will be $6 \times 3=18$ beads". Participant A15 was asked to express the pattern rule in the algebraic expression $\mathrm{T}_{n}=6 n$, and responded:

Joana will need 17 flowers with 102 white beads, I was counting how many white beads for the second flower, and I was looking for a rule that would work therefore I multiplied six which is the white beads by the number of flowers.

Participant A15 responded on the IP2 and used direct proportion. Participant A15 focused on taking information directly from the data by reading the data with little understanding. This finding is similar to Barbosa et al. (2012) revealing that learners using direct proportional strategy are not able to find adequate general rules, because they have difficulties in finding a functional relationship between the data and the mathematical operations. Participant A15 might have used direct proportional strategy, as he/she was unable to find an adequate general rule, and, thus had trouble in finding a functional relationship between the number of beads and the number of flowers.

Participant B2 implemented the solution in a manner that did not directly address the problem statement as he/she used an incorrect strategy for implementing the decreasing pattern. Participant B2 could hardly apply a mathematical operation, which was also evident in the analysis of his/her written activity. In the case of -2 (-3), Participant B2 confessed:

I added 2 and 3 to give me 5 but know that the common difference of the sequence was 3 so I 
ignored the subtraction sign and the negative sign added 2 and 3 to give me the next term.

However, Participant B2 could connect successive independent values in the problem, which, according to Lannin et al. (2006), shows that he/she used a recursive technique. This finding is in agreement with Akkan (2013) claiming that most learners who use the recursive strategy to generalise patterns are able to find near terms accurately, but are unable to implement a model to find later terms in a sequence. Although Participant B2 focused on the relationship between the common difference and the consecutive number, he/she could have made an error on the mathematical operation, as he/she might have been unable to find near terms accurately or to implement a model to find the next term in the sequence.

Participant C5 responded three times on IP3. Participant C5 could construct a mathematical model and use it to generalise a pattern. Specifically, Participant C5 could show how the number of a previous term depends on the number of the next term, thus establishing the relationship between terms and using this relationship to express the general formula of the pattern. Participant C5 processed knowledge about the obtained formula and, therefore, used a recursive strategy to generalise the pattern. Participant C5 used the common difference between two consecutive terms of the sequence to solve the questions posed. This finding corresponds with Barbosa et al. (2012) arguing that, learners use a recursive strategy correctly, if they can extend the sequence and generate the general formula using the common difference, but when using multiples of the common difference without final adjustment, it results in inaccurate answers. Participant C5 reached the IP3 and might have used a recursive strategy. $\mathrm{He} /$ she explained that each flower shares two white beads to have a complete flower and four white beads should be added to the end of each prior flower to create a new flower, which is the common difference, and then two beads should be added to give a complete flower.

\section{Strategies to Solve Number-Pattern Problems}

The findings from the analysis of the written activities and the interviews revealed four strategies to solve number-pattern problems, namely (1) counting; (2) recursive strategy; (3) direct proportion; (4) mental image representation; and (5) mental modal representation.

In the DP, the counting strategy was preferred in near generalisation, while the recursive strategy was favoured for far generalisation. The participants that used the counting strategy were unable to give a general rule for far generalisation. The majority of the participants applied this strategy in their execution of numerical linear patterns as compared to the pictorial patterns. The participants who used the counting strategy could not recognise a pattern for far generalisation. However, they were successful in observing the sequence of numbers by completing the pattern indicating the next terms or elements. This finding shows that these participants were able to use correct decoding of numbers for near generalisation when given a number pattern. This finding is in agreement with Becker and Rivera (2005, p.128) indicating that learners "who fail to provide the general formulae tend to start out with counting strategies; however, they lack the flexibility to try other approaches and see possible connections between different forms of representation and generalisation strategies".

The participants, who used the recursive strategy, were able to decode the problem statement in the DP, to use a mental model of the problem during the RP, and to proceed to PP by indicating the correct general rule of the problem. This finding is similar to Akkan (2013) revealing that most of the learners who use the recursive strategy are able to find near and far terms accurately in a pattern sequence.

The direct proportional strategy was evident in the $\mathrm{RP}$, PP and the IP. During the RP, the majority of participants used proportional representation by focusing on given or apparent information and prioritised manipulation of equations of common difference and multiplication (rote memorisation) when finding the $n^{\text {th }}$ term. During the PP, the participants did not show a strong visual image of the situation, but only focused on the numbers given in the problem. Those participants who attempted to generalise the problem, began with an incorrect drawing and directly counted the numbers from a pattern. They multiplied the numbers to generalise the sequence. During the IP, the participants directly picked up numbers from the given problems and applied multiplication to those numbers. They failed to obtain the correct answer and showed a lack of representing, processing and implementation. They did not understand the information within a given problem statement in order to solve the problem. This finding concurs with Barbosa et al. (2012) indicating that learners who use the direct proportional strategy fail to make a final adjustment based on the context of the problem.

The mental image representation strategy was evident in the RP. The few participants who used mental model representation, focused on comprehending the pattern and the mathematics concepts in the problem, and dealt with equations by including diagrammatic representations in their solutions. While they could provide reasons for the problem, they failed to handle the mathematical operations. This finding is similar to Ibrahim and Rebello (2013) revealing that many learners, who can construct mental images, deal with visual descriptions in isolation. 


\section{CONCLUSION}

The solving of number-pattern problems is often regarded as a difficult topic (Boonen et al., 2013; Jupri \& Drijvers, 2016) and many learners use inappropriate strategies to solve number-pattern problems. Learners can often not easily identify number patterns embedded in problems as they lack knowledge of appropriate mathematical concepts. Therefore, this study investigated Grade 9 mathematics learners' strategies in solving number-pattern problems. Four strategies to solve number-pattern problems were identified, namely (1) direct counting, (2) direct proportion, (3) recursive strategy, and (4) mental image representation. Both direct counting and the recursive strategy are evident during the DP. The competency of decoding is demonstrated when learners recognise and find a pattern in both the near and far position. The strategy of direct proportion is evident in the PP and the IP. Processing occurs when learners can create a general formula for the sequence, for example a mathematical model. The strategy of mental image representation transpires during the RP when learners state the problem in a language they can understand and are able to represent by means of a mental model.

Many teachers and instructional leaders all over the world need guidance pertaining to strategies to solve number patterns. Therefore, the authors foreground some suggestions. First, to develop the skill of decoding, it is recommended that learners should be provided with content knowledge of patterns. Teachers should use strategies that assist learners to use symbolic algebraic expressions and to generalise the sequence. Learners should be developed to use a recursive generalisation. Teachers need to inform learners on how to find the value of a term by giving the value of the preceding term. It is important that learners should be asked to explain their thinking. Having them describe their reasoning can also help them realise that often there is more than one way to look at a pattern.

Secondly, to acquire the skill of representing, it is suggested that learners should be guided on how to construct mental models, which will provide them with opportunities to use algebra to prove conjectures or rectify different solutions or formulae for number patterns. By drawing learners' attention to the relationships between the physical representation of a pattern and its symbolic expression, learners can be led to recognise that different symbolic expressions may represent the same physical situation.

Thirdly, to foster the skill of processing, it is proposed that learners should be assisted to develop accurate concepts on number pattern. The concept of algebraic expressions, to form equations to solve number patterns problems, is a powerful concept of elementary algebraic reasoning. Teachers should not only assist learners in using algebraic expressions, but also provide them with strategies to manipulate symbols in number patterns. Teachers should provide learners with content knowledge of number patterns, for example, the strategies that can be used in both numbers and pictorial patterns.

Finally, to develop the skill of implementing, it is advised that teachers should assist learners to focus on the relationship between the data in a problem and the mathematical operation. Learners should be guided to find and to discover a pattern, and to generalise and to implement a correct mathematical model. Thus, learners should be encouraged to reason about the strategies they use when solving number pattern problems.

Observations could have provided additional data on learners' thinking processes in solving problems. Due to the contextual nature of this study, the findings cannot be generalised. However, this study may be extended across different phases and to larger sample sizes. Further research may establish the relationship between learners' strategies in solving number-pattern problems and those of teachers teaching the topic.

The study contributes by identifying different strategies learners use in solving number-pattern problems, but also inform teachers about learners' interpretations of the strategies they utilise during the different phases of problem solving. The use of the PS conceptual framework of Singer and Voica (2013) as a tool to analyse the strategies learners employ to learn number-pattern problems is novel. The study also complements the limited research in South Africa on problem solving, specifically pertaining to number patterns. This study may sensitise teachers to introduce counting, direct proportion, recursive strategies, and mental image representation to learners when solving number-pattern problems.

\section{ACKNOWLEDGEMENT}

We are grateful to the three principals who allowed us to conduct the study in their schools and all the learners who participated in the study.

\section{REFERENCES}

Ahmad, A., Tarmizi, R. A., \& Mawawi, M. (2010). Visual representations in mathematical word problem solving among Form Four students in Malacca. Procedia - Social and Behavioral Sciences, 8, 356-361. https:// doi.org/10.1016/j.sbspro.2010.12.050

Akkan, Y. (2013). Comparison of 6th-8th graders' efficiencies, strategies and representations regarding generalization patterns. Bolema: Boletim de Educação Matemática, 27(47), 703-732. https:/ / doi.org/10.1590/S0103-636X201300040000

Anderson, J. (2009). Mathematics Curriculum Development and the Role of Problem Solving. 2009 National Biennial Conference of the Australian 
Curriculum Studies Association: Curriculum, 1-8. Retrieved from https:/ / www.acsa.edu.au/pages/ images/JudyAnderson-MathematicsCurriculum Development.pdf

Barbosa, A., Vale, I., \& Palhares, P. (2012). Pattern Tasks: Thinking processes used by 6th grade students. Revista Latinoamericana de Investigación En Matemática Educativa, 15(3), 273-293. Retrieved from https:/ / www.redalyc.org/jatsRepo/335/3352457 9002/html/index.html

Becker, J. R., \& Rivera, F. (2005). Generalization strategies of beginning high school algebra students. In H. Chick \& J. L. Vincent (Eds.), Proceeding of the 29th Conference of the International Group for the Psychology of Mathematics Education (pp. 121-128). Melbourne, Australia: University of Melbourne. Retrieved from https://www.emis.de / proceedings/PME29/PME29RRPapers/PME29V ol4RossiBeckerRivera.pdf

Biddlecomb, B., \& Carr, M. (2011). A longitudinal study of the development of mathematics strategies and underlying counting schemes. International Journal of Science and Mathematics Education, 9(1), 1-24. https: / / doi.org/10.1007/s10763-010-9202-y

Boonen, A. J. H., van der Schoot, M., van Wesel, F., de Vries, M. H., \& Jolles, J. (2013). What underlies successful word problem solving? A path analysis in sixth grade students. Contemporary Educational Psychology, 38(3), 271-279. https://doi.org/10.1016 /j.cedpsych.2013.05.001

Clement, J. J. (2008). Does decoding increase word problem solving skills? (University of Nebraska-Lincoln, Lincoln, NE). Retrieved from https://digitalcom mons.unl.edu/mathmidactionresearch/32/

Department of Basic Education. (2011). Curriculum and Assessment Policy Statement: Further Education and Training Phase. Grades 10-12 Mathematical Literacy. Retrieved from https://www.education.gov. za/Portals/0/CD/NationalCurriculumStatements andVocational/CAPSFET_MATHEMATICALLIT ERACY_GR10-12_Web_DDA9.pdf?ver=2015-0127-154330-293

Duru, A., Peker, M., Bozkurt, E., Akgün, L., \& Bayrakdar, Z. (2011). Pre-service primary school teachers' preference of the problem solving strategies for word problems. Procedia - Social and Behavioral Sciences, 15, 3463-3468. https://doi.org/10.1016/ j.sbspro.2011.04.319

Garcia-Cruz, J. A. A., \& Martinon, A. (1997). Actions and invariant in schemata in linear generalising problems. In E. Pehkonen (Ed.), Proceedings of the 21st Conference of the International Group for the Psychology of Mathematics Education (pp. 289-286). Retrieved from https://jagcruz.webs.ull.es/ Articulos/pme97.pdf
Govender, V. G. (2012). Using the South African mathematics challenge to develop pre-service mathematics teachers' problem-solving abilities. In M. Lebitso, \& A. Maclean (Eds.), 20th Annual National Congress of the Association for Mathematics Education of South Africa (AMESA): Demystifying mathematics (pp. 87-104). Kimberley, South Africa: AMESA. Retrieved from http://www.amesa. org.za/AMESA2014/Proceedings/images/Front \%20Matter\%202014\%20AMESA.pdf

Güner, P., Ersoy, E., \& Temiz, T. (2013). 7th and 8th Grade students' generalization strategies of patterns. International Journal of Global Education, 2(4), 39-54. Retrieved from http:/ / www.ijtase.net/ ojs/index.php/ijge/article/view/284/348

Ibrahim, B., \& Rebello, N. S. (2013). Role of mental representations in problem solving: Students' approaches to nondirected tasks. Physical Review Special Topics - Physics Education Research, 9(2), 1-17. https:/ / doi.org/10.1103/PhysRevSTPER.9.020106

Jan, S., \& Rodrigues, S. (2012). Students' difficulties in comprehending mathematical word problems in English language learning contexts. International Researchers, 1(3), 152-160. Retrieved from http:/ / www.iresearcher.org/150-160 Salma.pdf

Jupri, A., \& Drijvers, P. (2016). Student Difficulties in Mathematizing Word Problems in Algebra. EURASIA Journal of Mathematics, Science $\mathcal{E}$ Technology Education, 12(10), 2481-2502. https:/ / doi.org/10.12973/eurasia.2016.1299a

Jurdak, M. E., \& El Mouhayar, R. R. (2014). Trends in the development of student level of reasoning in pattern generalization tasks across grade level. Educational Studies in Mathematics, 85(1), 75-92. https:/ / doi.org/10.1007/s10649-013-9494-2

Killen, R. (2007). Teaching strategies for outcomes-based education (2nd ed.). Cape Town: Juta.

Lannin, J., Barker, D., \& Townsend, B. (2006). Algebraic generalisation strategies: Factors influencing student strategy selection. Mathematics Education Research Journal, 18(3), 3-28. https://doi.org/ 10.1007/BF03217440

Liljedahl, P. (2004). Repeating pattern or number pattern: The distinction is blurred. Focus on Learning Problems in Mathematics, 26(3), 24-42. Retrieved from http://www.peterliljedahl.com/wp-content / uploads/JA-FLPM-2004.pdf

Liljedahl, P., Santos-Trigo, M., Malaspina, U., \& Bruder, R. (2016). Problem Solving in Mathematics Education. In Problem Solving in Mathematics Education. ICME-13 Topical Surveys. (pp. 1-39). https:/ / doi.org/10.1007/978-3-319-40730-2_1

Mahlobo, N., \& Nthombela, R. (2014). How I teach patterns in a Grade one class. In M. Lebitso \& A. Maclean (Eds.), The 20th Annual National Congress of 
the Association for Mathematics of South Africa: Demystifying mathematics (pp. 186-189). Retrieved from http://www.amesa.org.za/AMESA2014/Pr oceedings/images/FrontMatter2014AMESA.pdf

Maluleka, B. K. (2013). Improving Grade 9 learners' mathematical processes of solving word problems. (Master's dissertation). University of Limpopo, Mankweng, South Africa. Retrieved from http:/ / hdl.handle.net/10386/965

Schumacher, R. F., \& Fuchs, L. S. (2012). Does understanding relational terminology mediate effects of intervention on compare word problems? Journal of Experimental Child Psychology, 111(4), 607628. https:/ / doi.org/10.1016/j.jecp.2011.12.001
Singer, F. M., \& Voica, C. (2013). A problem-solving conceptual framework and its implications in designing problem-posing tasks. Educational Studies in Mathematics, 83(1), 9-26. https:/ / doi.org/ 10.1007/s10649-012-9422-x

Tambychik, T., \& Meerah, T. S. M. (2010). Students' Difficulties in Mathematics Problem-Solving: What do they Say? Procedia - Social and Behavioral Sciences, 8, 142-151. https://doi.org/10.1016/j.sbspro.2010. 12.020

Van Staden, S., \& Motsamai, P. (2017). Differences in the quality of school-based assessment: Evidence in Grade 9 mathematics achievement. Pythagoras, 38(1), a367. https://doi.org/10.4102/pythagoras. v38i1.367

\section{http://www.ejmste.com}

